\title{
Caracterização das práticas de hidratação em karatecas do estado de Minas Gerais
}

\author{
Artigo Original
}

\section{Igor Surian de Souza Brito}

Graduando em Educação Física pela Universidade Federal de Viçosa/MG igorsurian@yahoo.com.br

\section{Ciro José Brito}

Mestrando em Ciência da Nutrição e Saúde da Universidade Federal de Viçosa/MG cibrito@argentina.com
Sabrina Pinheiro Fabrini - CRN 100678-9/MG

Nutricionista graduada pela Universidade Federal de Viçosa/MG Especialista em Nutrição Esportiva/Universidade Gama Filho sapfabrini@yahoo.com.br

João Carlos Bouzas Marins - CREF 3976-G/MG

Professor adjunto do Dept ${ }^{a}$ de Educação Física da Universidade Federal de Viçosa/MG jcbouzas@ufv.br

BRITO, I. S. S.; BRITO, C. J; FABRINI, S. P.; MARINS, J. C. B. Caracterização das práticas de hidratação em karatecas do estado de Minas Gerais. Fitness \& Performance Journal, v. 5, n 1, p. 24 - 30, 2006.

RESUMO: Este trabalho teve como objetivo verificar os conhecimentos sobre hidratação de um grupo de karatecas do estado de Minas Gerais. Foram avaliados 135 atletas (97 homens e 35 mulheres). Utilizou-se um questionário padronizado com 18 perguntas objetivas auto-administrativo sobre hidratação. Os resultados indicam que aproximadamente $50 \%$ dos atletas se hidratam durante os treinamentos ou competições. A solução mais consumida para hidratação é água. Do total da amostra $45,19 \%$ nunca se pesam antes dos treinamentos ou competições. As manifestações fisiológicas mais prevalentes durante um treinamento ou competição foram: dificuldade de concentração $(38,52 \%)$, sede muito intensa $(35,56 \%)$, sensação de perda de força $(34,81 \%)$ e câimbras $(20,74 \%)$. Aproximadamente $40 \%$ apresentam uma idéia correta sobre a freqüência e volume de hidratação. A maioria dos atletas $(60,47 \%)$ nunca foi orientada quanto à melhor maneira de se hidratar. Apesar do baixo consumo, 63,7\% dos atletas sabem a função correta de uma solução carboidratada. Conclui-se que os karatecas avaliados apresentam poucos conhecimentos sobre hidratação e apresentam hábitos inadequados de reposição hídrica.

Palavras-chave: karatê, treinamento, hidratação, desidratação, bebida carboidratada, desempenho.

Endereço para correspondência: R Universidade Federal de Viçosa Departamento de Educação Física - LAPEH A/C Prof. Dr. João Carlos Bouzas MARINS - CEP: 36570-000 Viçosa/MG - Brazil 


\section{Hydration practices characteristics of karate fighters from Minas Gerais state}

The hydration state may be determinant to sport triumph. The aim of this study was to verify the knowledge on hydration of a group of karate athletes from the state of Minas Gerais. One hundred and thirty five athletes ( 97 male and 35 female) were evaluated. It was used a standardized questionnaire with 18 questions on hydration. The results showed that around $50 \%$ of the athletes hydrate during either training sessions or competitions, with greater prevalence before, during and after training sessions compared with competitions. Nevertheless, $4.41 \%$ of the subjects did declare never had used hydration during training sessions. The most consumed solution to hydration amongst the subjects was water. The majority of the athletes $(66.42 \%)$ was used do consume liquid before the sensation of thirst. Less than a half of the subjects (45.19\%) did not use to weigh before training sessions or competitions. The most prevalent physiologic manifestations during training sessions or competitions were difficulty of concentration (38.52\%), very intense thirst (35.56\%), loss of strength sensation (34.81\%) and cramp (20.74\%). Around $40 \%$ of the athletes exhibited a right idea about the frequency and volume of hydration. The majority of the subjects $(60.47 \%)$ never had been told about the best way of hydration, and amongst those who had such information it came from coaches (46\%), physicians (22\%) and nutritionists (20\%). Despite the low consumption, $63.7 \%$ of the subjects knew the role of a carbohydrate solution. It was concluded that karate athletes showed pour knowledge on hydration and presented inadequate habits of fluid replacement. It suggests that information about adequate habits of hydration is needed.

Keywords: karate, training, hydration, dehydration, sport drink, performance.
Caracterización sobre las practicas de hidratación em luchadores de karate en la región de Minas Gerais

La condición de hidratación puede determinar el éxito en una competición. Este trabajo tuvo como objetivo identificar el grado de conocimiento sobre hidratación de un grupo de luchadores de karate de la región de "Minas Gerais". Fueron evaluados 135 luchadores (97 hombres y 35 mujeres). Se empleó una encuesta conteniendo 18 preguntas objetivas sobre hidratación. Los resultados indican que aproximadamente $50 \%$ de los deportistas hidrataban durante los entrenamientos o competiciones, con mayor frecuencia de hidratación ocurriendo antes, durante y después de los entrenamientos frente a competiciones. Sin embargo 4,41\% respondieron que nunca hidratan durante los entrenamientos. La bebida más consumida fue el agua. La mayor parte de los deportistas $(66,42 \%)$ consumen líquidos antes de sentir sed. Del total de la muestra 45,19\% nunca se pesan antes de los entrenamientos o competiciones. Las señales fisiológicas más frecuentes durante un entrenamiento o competición fueran: dificultad de concentración $(38,52 \%)$, sed muy intensa $(35,56 \%)$, sensación de pérdida de fuerza $(34,81 \%)$ y calambres $(20,74 \%)$. Aproximadamente $40 \%$ presentan una idea correcta sobre la frecuencia y volumen de hidratación. La mayor parte de los deportistas $(60,47 \%)$ nunca fueron orientados sobre la mejor manera de hidratar, entre los que ya recibieron orientaciones las fuentes más comunes fueron el entrenador (46\%), el médico (22\%) y el nutricionista (20\%). Mismo con un bajo consumo $63,7 \%$ de los deportistas conocen la función correcta de una bebida deportiva. Se concluye que los luchadores de karate evaluados presentan poco conocimiento sobre hidratación, además de presentaren hábitos inadecuados de reposición hídrica, sugiriendo la creación de campañas nutricionales para que informen a los deportistas sobre a importancia de mantengan hábitos adecuados de hidratación.

Palabras clave: karate, entrenamiento, hidratación, deshidratación, bebida carbohidratada, desempeño.

\section{INTRODUÇÃO}

Modalidades desportivas de alto rendimento requerem grande dedicação por parte dos atletas para que os resultados ocorram ao final do período de competição. Aliado ao esforço dos atletas é necessário a preparação adequada dos treinadores e de todos os demais membros da equipe que acompanham o desportista, para que no final, a soma de todas as forças alcancem os resultados planejados.

O caratê é uma modalidade que se caracteriza como anaeróbica intermitente, em que a força explosiva e a flexibilidade de membros superiores e inferiores são importantes para o desempenho do atleta. Segundo Mori et al. (2002), o caratê é uma modalidade que requer reações rápidas, para que o lutador não seja acuado pelo adversário, além de exigir alta capacidade de velocidade e força para golpear o oponente.

No caratê competitivo muitas vezes o nível técnico dos lutadores se equivale, fazendo com que uma competição seja definida por detalhes. A nutrição pode ser este fator decisivo em uma competição. Para Guerra (2004), uma alimentação balanceada se faz necessário para que o atleta possa maximizar seu desempenho. De acordo com o consenso emitido pela ADA/ ACSM/DC (2000), os atletas, independentemente do sexo, podem ter necessidades nutricionais especiais, devido às considerações especiais de cada modalidade.

Dentro da nutrição esportiva, a hidratação tem sido amplamente estudada como fator delimitador do desempenho desportivo, quando o atleta entra em um campeonato em estado de euhidratação, ele pode desenvolver o melhor de sua capacidade técnica, entretanto um suporte hídrico inadequado pode afetar negativamente a performance do atleta (MARINS et al., 2000).

A hidratação esta intimamente ligada aos esportes de combate categorizados por peso. No caratê, assim como no boxe, luta olímpica e judô, existe atletas que se utilizam de métodos para perda de peso rápida através da desidratação. Sobre as conseqüências fisiológicas da desidratação para o exercício físico, Marins et al. (2000) publicou uma revisão, onde são apresentadas 21 manifestações fisiológicas negativas para o atleta advindas da desidratação.

Diversos estudos foram elaborados para identificar o nível de conhecimento e práticas habituais de atletas sobre hidratação, como, por exemplo, os trabalhos de Brito e Marins (2001) com judocas, Marins et al. (2004) com ciclistas, triatletas e corredores, Ferreira e Marins (2002b) com jogadores de futebol. Especificamente, a relação de perda hídrica em modalidades de luta foi alvo de estudos por Ohta et al. (2002) e Suzuki et al. (2003) em judocas, Hall e Lane (2001) em boxeadores, Roemmich e Sinning (1997) e Landers et al. (2001) em luta olímpica.

Apesar de a desidratação estar presente diariamente na modalidade de caratê, não se encontram trabalhos científicos sobre este tema. Tomando como referência uma busca realizada na base de dados do MedLine em 28/04/2005, foram encontrados 
mais de 350 artigos sobre caratê, entretanto nenhum deles sobre a temática da hidratação. Dessa forma, este trabalho pode ser considerado como pioneiro ao examinar os hábitos de hidratação de karatecas.

Conhecer os hábitos de hidratação de karatecas é fundamental para orientar os treinadores e atletas sobre as necessidades de reposição hídrica da modalidade. A partir dessas informações, os treinadores podem estabelecer estratégias para consumo de líquidos antes, durante e depois dos treinamentos ou competições.

Assim sendo, o objetivo deste estudo foi avaliar os hábitos de hidratação de um grupo de karatecas.

\section{METODOLOGIA}

Instrumento: Empregou-se uma metodologia exploratória através de uma pesquisa do tipo descritiva, utilizando um questionário padronizado com 18 perguntas objetivas, auto-administrativo, já aplicado anteriormente em maratonistas (MARINS et al., 1999 ${ }^{\circ}$ ), triatletas (MARINS et al., 1999b), judocas (BRITO e MARINS, 2001), universitários (FERREIRA e MARINS, 2002a) e jogadores de futebol (FERREIRA e MARINS, 2002b). No Anexo I encontra-se o modelo do questionário aplicado aos atletas.

Características da amostra: Foram entrevistados 135 atletas (97 homens e 38 mulheres). A amostra foi selecionada de maneira aleatória. Os atletas apresentavam uma média de idade de 22,81 $\pm 6,03$ anos, experiência prévia de competições na modalidade de 6,64 $\pm 6,38$ anos e peso corporal de 64,11 kg $\pm 11,47$.

Coleta de dados: A coleta de dados foi realizada em equipes de caratê filiadas a FMK (Federação Mineira de Karate). A coleta compreendeu o período de março a abril de 2005. Os atletas entrevistados pertenciam a academias das seguintes cidades: Belo Horizonte, Ipatinga, Juiz de Fora, Ubá, Uberlândia, Viçosa, todas localizadas no estado de Minas Gerais.

Tratamento estatístico: Para análise estatística utilizou-se a distribuição percentual obtida em cada resposta, sendo descartadas as questões não respondidas de cada questionário.

\section{Resultados e discussão}

Os principais resultados do estudo são apresentados a seguir, segundo a ordem das perguntas respondidas pelos atletas.

Sobre o hábito de se hidratarem durante os treinamentos e competições, $47,06 \%$ da amostra disseram que sempre se hidratam durante os treinamentos e 53,13\%, durante as competições. Esse fato se mostra preocupante, pois aproximadamente metade dos entrevistados apresentou hábitos inadequados de hidratação; é recomendável que os atletas sempre se hidratem durante os treinamentos e competições (ACSM, 1996a; MARINS et al., 2000). Resultados similares foram encontrados por Brito e Marins (2001), em uma mostra de judocas $(53,96 \%$ e 54,34\% se hidratavam sempre durante os treinamentos e competições).

Um pequeno número de atletas relatou nunca se hidratar durante os treinamentos ou competições (4,41\% durante os treinamentos e 11,46\% em competições); dados alarmantes, uma vez que a desidratação tende a elevar a temperatura corporal (MARINS et al., 2000), o que pode reduzir a capacidade física do atleta, principalmente se ele está competindo em ambientes quentes (STRACHAN et al., 2005).

Mortes por desidratação aconteceram em esportes categorizados por peso em atletas que se preparavam para competições (CDC, 1998), o que torna fundamental o consumo de líquidos durante os treinamentos. Entretanto, a quantidade de líquidos a ser consumida durante o treinamento ou competição deve ser calculada de acordo com a perda do atleta, pois o excesso de água também pode ser prejudicial, podendo causar hiponatremia (ALMOND et al., 2005).

Em relação ao hábito de se hidratar durante os treinamentos ou competições, os resultados são apresentados na Figura 1.

De acordo com a Figura 1, os karatecas apresentam maior consumo de líquidos, antes durante e depois dos treinamentos, em relação às competições. Esse hábito pode interferir negativamente no desempenho dos atletas em competições, pois a estratégia de hidratação realizada na competição deve ser treinada previamente (BRITO e MARINS, 2001 ; MARINS et al., 2004). Possivelmente, o maior consumo de líquidos durante os treinamentos se deva ao tempo dedicado a cada atividade, pois os treinamentos facilmente ultrapassam os 100 minutos de duração; por outro lado, as lutas de competição, quando não são decididas pela aplicação de uma técnica perfeita, não ultrapassam 3 minutos (LEMOS, 2001), fazendo com que as demandas hídricas em cada uma das atividades sejam diferentes.

Ainda sobre a Figura 1, observa-se um maior consumo de líquidos após os treinamentos ou competições. De acordo com o consenso coletivo estabelecido pelo American Dietetic Association, - American College of Sports Medicine e o Dietitians of Canada, para se iniciar o exercício bem hidratado deve-se consumir entre 400 e $600 \mathrm{ml}$ de líquido de 2 a 3 horas antes do exercício. Para manter a homeostasia durante o exercício, entre 150 e $350 \mathrm{ml} \mathrm{a}$ cada 15-20 minutos. Após o exercício, os atletas devem consumir até $150 \%$ do peso corporal perdido durante a atividade (ADA) ACSM/DC, 2000).

Do total de atletas, aproximadamente $50 \%$ se preocupam com o tipo de solução, entretanto para os demais não faz diferença se hidratarem com água ou solução carboidratada. Estudos têm demonstrado vantagens do consumo de isotônico em relação à água (NIEUWENHOVEN et al., 2000; DAVIS et al., 1997). Para maior aprofundamento sobre a composição da solução de reidratação, perguntou-se aos atletas qual tipo de solução eles consomem em cada momento, os resultados são apresentados na Figura 2.

Observa-se claramente um maior consumo de água em relação ao consumo de bebida carboidratada. Para Marins (1996), o consumo de uma bebida carboidratada auxilia na manutenção da glicemia sanguínea, adia o aparecimento da fadiga e apresenta rápida absorção intestinal. Assim sendo, um aumento no consumo de bebidas carboidratadas seria recomendado para melhorar a qualidade do treinamento. Como já citado anteriormente, o caratê é uma modalidade em que velocidade e força são importantes para o êxito do atleta, assim sendo, o metabolismo glicolítico será solicitado durante grande parte do treinamento ou competição. $\bigcirc$ consumo de uma solução carboidratada 
seria altamente recomendado, uma vez que atividades intensas demandam em grande parte do catabolismo de carboidratos (SAUNDERS et al., 2001).

Após o exercício, apenas 16,3\% dos karatecas repõem energia através de bebidas carboidratadas. Recomenda-se um maior consumo de carboidratos após o exercício para acelerar a reposição do glicogênio muscular e hepático (MARINS et al., 2004). Degoutte et al. (2003) em seu estudo com judocas, observou que o baixo consumo de carboidratos está relacionado à baixa ressíntese do glicogênio.

Sobre o momento em que devem se hidratar, 66,42\% dos avaliados afirmam que devem consumir líquidos antes de sentirem sede, $21,9 \%$ somente depois de sentirem sede e 11,68\% após sentirem muita sede. Os treinadores devem estimular os atletas a se hidratarem antes de sentirem sede, de acordo com Greenleaf et al. (1992), o sinal de sede aparece quando o atleta já apresenta $2 \%$ de desidratação. Este grau de desidratação leva a reações fisiológicas negativas, como redução em 10\% do Vo2máx, redução da taxa de esvaziamento gástrico e aumento da temperatura interna (MARINS et al. 2000).

Em relação a qual tipo de solução preferem para se hidratar, os resultados estão apresentados na Figura 3.

De acordo com os dados apresentados acima, a solução mais consumida como agente reidratante é a água; resultados similares foram encontrados por Marins et al. (2004), ao avaliar corredores triatletas e ciclistas, e por Ferreira e Marins (2002a) em atletas universitários. Nota-se uma carência de orientações adequadas sobre hidratação aos karatecas, pois tornou-se evidente marcada preferência para o consumo de bebidas impróprias à prática esportiva, como Coca-cola ${ }^{\circledR}(11,1 \%)$, cerveja $(8,89 \%)$ e café $(8,89 \%)$, em detrimento do consumo de bebida carboidratada (5,49\%). De acordo com Marins et al. (2004), o consumo de bebidas gasosas durante o exercício causa desconforto gástrico. O consumo de bebida alcoólica resultará em desidratação, um efeito contrário ao que se busca, ao ingerir um líquido durante o exercício (CASA et al., 2000). O consumo de café tem sido recomendado devido à ação ergogênica da cafeína (WALTON et al., 2002), entretanto sua ingestão como solução reidratante não é recomendada (BRITO e MARINS, 2001).

Apesar do baixo consumo de bebida carboidratada, os atletas apresentam um consumo moderado de outras fontes líquidas que fornecem carboidratos, como refrescos $(20 \%)$ e sucos naturais $(47,41 \%)$. De acordo com Brito e Marins (2001), parte do carboidrato pode ser reposto na forma de sucos ou refrescos. Esta reposição pode ser feita principalmente após o exercício, quando os receptores de insulina estão mais ativos, o que facilita a reposição do glicogênio (GUERRA, 2004).

Quando perguntados sobre o tipo de solução que conhecem, as marcas de bebidas mais populares foram Gatorade $\AA(86,67 \%)$, Energil $C \circledR(45,19 \%)$ e Red Bull $\AA(44,44 \%)$. Entretanto, quando questionados sobre qual a marca de preferência 77,04\% afirmaram preferir a marca Gatorade ${ }^{\circledR}$, já os demais produtos apresentaram uma preferência muito inferior entre os karatecas (6,67\% para o Energil C $\AA$ e 5,93\% para o Red Bull $®)$. Esta baixa preferência pelo Red Bull $\AA^{\circledR}$ se mostra como um fator positivo, pois este energético, apesar de bem conhecido, não deve ser
Figura 1 - Consumo de líquidos antes, durante e após os treinamentose competições em karatecas

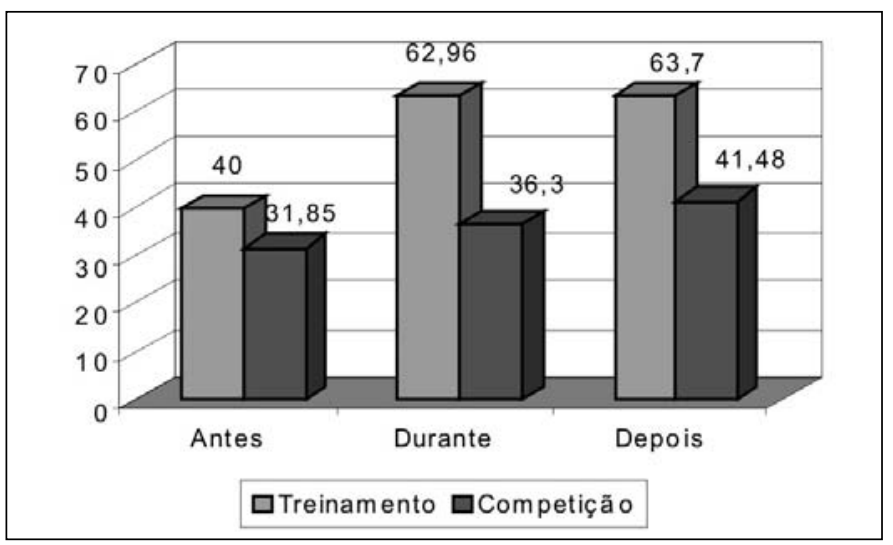

Figura 2 - Tipo de solução consumida a cada momento por karatecas

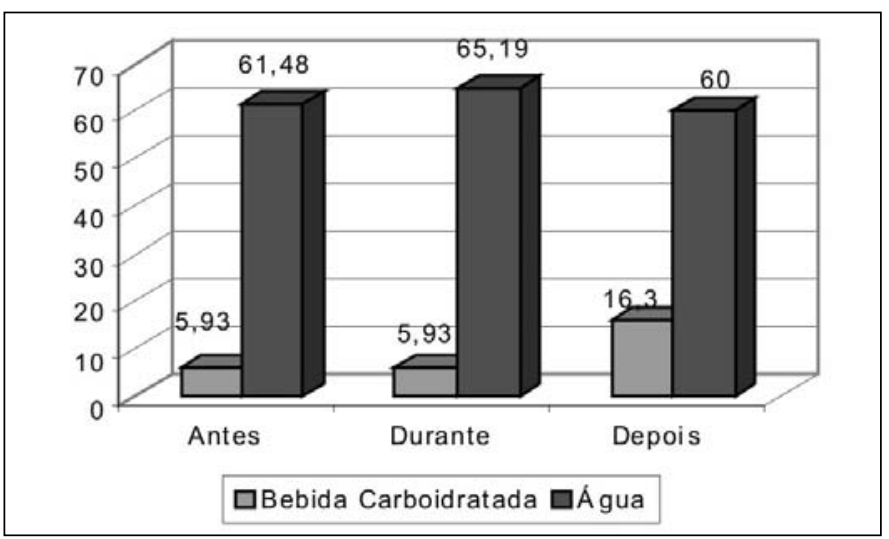

Figura 3 - Soluções consumidas para reidratação

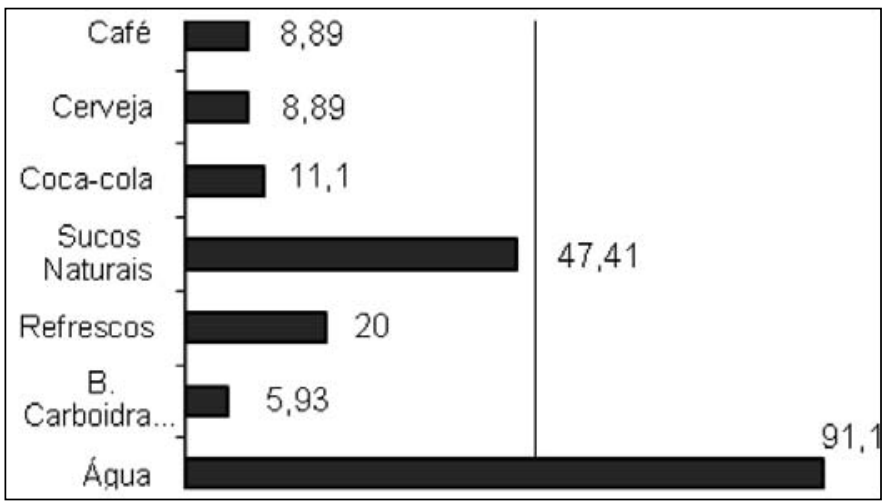

consumido durante o exercício por não se tratar de uma bebida desportiva (BONCl, 2002).

Os sabores de maior preferência são: laranja (31,85\%), frutas cítricas $(22,22 \%)$, maracujá $(21,48 \%)$ e limão $(15,56 \%)$. Esses resultados diferem da preferência apontada pelos judocas, de acordo com a qual o sabor mais apreciado é tangerina $(36,63 \%)$ (BRITO e MARINS, 2001). O sabor da bebida influencia na quantidade de líquidos a ser ingerido pelo atleta (ADA/ACSM/DC, 2000), além disso, o ACSM (1996a) observa que a flavorização das soluções de reidratação aumentam o consumo voluntário em atletas.

Quando perguntados se adotam hábitos de hidratação distintos em determinadas épocas do ano, verificamos que $46,67 \%$ se 
preocupam mais no verão, e para 43,7\%, independe da estação. Marins et al. (2004) verificaram em atletas europeus uma maior preocupação em se hidratar no verão; tal comportamento se explica pelas diferenças climáticas, pois na Europa as estações do ano são mais bem definidas, ao contrário do Brasil, onde a temperatura não varia tanto ao longo do ano, e a maioria dos atletas não apresenta maior preocupação com a hidratação em um período em relação a outro. Resultados similares foram encontrados por Brito e Marins (2001) em judocas de Minas Gerais.

Em relação ao hábito de se pesarem antes e depois de cada treinamento, encontramos um dado interessante, a maior parte da amostra $(45,19 \%)$ nunca se pesa, seguida dos atletas que quase nunca se pesam (34,07\%). Do total da amostra, apenas 5 atletas se pesam antes e depois dos treinamentos. Esse é um comportamento totalmente inadequado, por se tratar de esporte categorizado por peso. Quando o atleta não se pesa, dificilmente vai conhecer sua necessidade de reposição hídrica durante o treinamento. Além disso, quando o atleta não controla o peso ao longo do período de preparação, ele pode necessitar de uma estratégia aguda para redução de peso no período pré-competição. O ACSM (1996b) desencoraja qualquer tipo de estratégia de redução brusca de peso, pois isso coloca em risco a saúde do atleta.

Quando questionados sobre algum tipo de sintoma apresentado durante os treinos ou competições, as principais manifestações fisiológicas observadas foram: dificuldade de concentração $(38,52 \%)$, sede muito intensa $(35,56 \%)$, sensação de perda de força $(34,81 \%)$ e câimbras $(20,74 \%)$. Como já citado anteriormente, a sensação de sede intensa é um sinal decorrente de uma desidratação superior a $2 \%$. A dificuldade de concentração e a sensação de perda de força talvez sejam decorrentes da hipoglicemia, demonstrando que o consumo de carboidratos durante o exercício é fundamental para a manutenção do desempenho no exercício e da atividade do Sistema Nervoso Central (WINNINCK et al., 2005). Segundo Greenleaf (1992), a dificuldade de concentração é um sinal fisiológico decorrente de uma desidratação superior a $5 \%$. $\bigcirc$ aparecimento de cãibras pode estar relacionado à perda excessiva do mineral sódio (MARINS, 2000), este é outro fator pelo qual se recomenda o consumo de soluções carboidratadas para atletas, uma vez que este mineral é reposto neste tipo de bebida.

Sobre a freqüência e volume de hidratação, 40,74\% afirmam que se deve beber $250 \mathrm{ml}$ a cada 15 minutos, o que está de acordo com as proposições do ACSM (1996a), por outro lado, 44,44\% dos karatecas responderam não ter idéia da quantidade e freqüência correta de hidratação, além disso, 11,28\% da amostra têm uma idéia incorreta sobre maneira correta de se hidratar. $\bigcirc$ que demonstra a importância de se estabelecer orientações de qualidade para o grupo avaliado. Valores similares a estes foram encontrados em judocas (BRITO e MARINS, 2001) e em ciclistas (MARINS et al., 2004).

Do total da amostra, $80 \%$ se preocupam com o tipo de roupa que utilizam além do quimono (obrigatório pela modalidade). Entre os que manifestaram preocupação, 58,33\% demonstraram preocupação com o tipo de tecido, 32,41\% com a quantidade de tecido e 12,03\% com a cor do tecido. A vestimenta utilizada na atividade física é fundamental para a termorregulação, pois esta pode facilitar ou dificultar a perda do calor corporal. A baixa preocupação com a cor do tecido pode impor um estresse térmico a mais para o atleta, uma vez que as cores mais escuras absorvem mais calor (MARINS et al., 2004). Os resultados foram similares aos encontrados em judocas $(17,4 \%)$ (BRITO e MARINS, 2001), corredores (8\%), triatletas (3\%) e ciclistas (3\%) (MARINS et al., 2004).

Em relação à temperatura do líquido utilizado para hidratação, $53,33 \%$ preferem a bebida moderadamente gelada, 37,04\% preferem temperatura normal e 6,67\%, extremamente gelada. A temperatura do líquido não irá interferir na capacidade de esvaziamento gástrico, dessa forma a temperatura ideal para a reposição hídrica é aquela que em que o atleta está acostumado (FERREIRA e MARINS, 2002a). Avaliando atletas de provas de resistência Marins et al. (2004) observaram que estes evitavam o consumo de bebidas extremamente geladas, pois lhes causava mal estar. $\bigcirc$ mesmo pode ser observado nos resultados do presente estudo.

Do total da amostra, 60,74\% nunca tiveram uma orientação sobre qual a melhor maneira de se hidratar, o que pode explicar os hábitos incorretos manifestados pelos karatecas avaliados. Dentre os que já tiveram algum tipo de orientação, a maioria obtém informações do treinador (46\%), nutricionista (22\%) e médico (20\%). Apesar do baixo número de atletas orientados, as fontes onde foram obtidas as informações parecem ser as mais confiáveis, entretanto Graves et al. (1991); Soper et al. (1992) ressaltam que, muitas vezes, treinadores apresentam idéias equivocadas sobre hidratação. Esse tipo de observação é fundamental, uma vez que os atletas tendem a confiar na orientação de seus treinadores (MARINS et al., 2004).

Por último, os atletas responderam o que acham ser a função da bebida carboidratada. A maioria dos atletas $(63,7 \%)$ respondeu corretamente, afirmando que uma bebida carboidratada hidrata, repõe eletrólitos e energia. Este dado apresenta um contra-senso entre o conhecimento e a prática da maioria dos karatecas avaliados, pois a maioria se hidrata com água, apesar de conhecer as funções de uma bebida carboidratada.

\section{CONCLUSÕES E RECOMENDAÇÕES}

De acordo com nossos resultados, a maioria dos karatecas avaliados não se hidrata corretamente, conforme as preconizações de entidades e especialistas no assunto. Uma vez que a desidratação pode interferir no desempenho do atleta, a incorporação de hábitos adequados para reposição de líquidos é fundamental para a performance desportiva.

Recomenda-se que as Federações responsáveis pela modalidade verifiquem se os resultados deste estudo se confirmam em outras regiões do Brasil. Será necessário o estabelecimento de campanhas de orientação a atletas e treinadores para que se adotem hábitos de reposição hídrica que atendam às necessidades da modalidade.

\section{BIBLIOGRAFIA}

American Dietetic Association, American College Sports Medicine, Dietitians of Canada. Joint Position Statement. Nutrition ad Athletic Performance. Med. Sci. Sports Exerc. 32(12):2130-2145, 2000. 
ACSM, Position stand. Exercise and fluid replacement, Med. Sci. Sports Exerc. 28 (1):i-vii, 1996a.

ACSM, Position stand. Weight loss in Wrestlers, Med. Sci. Sports Exerc. 28 (2):ix-xii, $1996 \mathrm{~b}$.

ALMOND C.S.D., et al. Hyponatremia among runners in the Boston Marathon. N Engl J. Med. 352:1550-6, 2005

$\mathrm{BONCl}$, L., "Energy" drinks: help, harm or hype?, www.gssiweb.com, 2002, data de acesso, 20/04/2005.

BRITO C.J., MARINS J.C.B. Hábitos de Hidratação em Judocas, Anais do XXIV Simpósio Internacional de Ciências do Esporte, 2001.

CDC. Centers for Disease Control and Prevention. Heat-Related Mortality - United State, 1997. JAMA 47:473-6, 1998

DAVIS, J.M.; JACKSON, D. A;. BROADWELL, M. S.; QUERI, J. L.; LAMBERT, C.L.; Carbohydrate drinks delay fatigue during intermittent, high-intensity cycling in active men and women. International Journal of Sports Nutrition 7:261-273, 1997.

DEGOUTTE, F; JOUANEL, P.; FILAIRE, E. Energy demands during a judo match and recovery Br. J. Sports Med. 37:245-9, 2003.

FERREIRA, F.G., MARINS, J.C.B. Hábitos e prática de hidratação dos atletas universitários da UFV. Rev. Min. Ed. Fís. 10(1):337, 2002a

FERREIRA, F.G., MARINS, J.C.B. Hábitos de hidratação de atletas de futebol júnior. Rev. Min. Ed. Fís. 10(1):338, 2002b.

GRAVES, K.; FARTHING, M.; SMITH, S.; TURCHI, J. Nutrition training, attitudes, knowledge recommendations, responsibility, and resources utilization of high school coaches and trainers. J. Am. Diet. Association 91:321-4, 1991.

GREENLEAF, J. Problem: thirst, drinking behavior, and voluntary dehydration, Med. Sci. Sports Exerc. 24 (6): 645-656, 1992.

GUERRA, I. Importância da alimentação e hidratação do atleta. R. Min. Educ. Fís 12(2):159-73, 2004

HALL, C.J.; LANE, A.M. Effects of rapid weight loss on mood and performance among amateur boxers. Br. J. Sports Med. 35:390-5, 2001

LANDERS, D.M.; ARENT, S.M.; LUTZ, R.S. Affect and cognitive performance in high school wrestlers undergoing rapid weight loss. J. Sports Exer. Psychol. 23:307-16, 2001.

LEMOS, E.D. La preparación física en karate: un primer enfoque. EFdeportes Revista Digital 34(7), 2001.

MARINS, J. C. B. Exercício físico e calor - Implicações fisiológicas e procedimentos de hidratação, Revista Brasileira de Atividade Física e Saúde, v.1 (3):26-38, 1996.
MARINS, J. C. B.; MARINS, N. M. O.; VILLEGAS, J.; ZAMORA, S. Hábitos de hidratación en un colectivo de corredores de maratón, Curso Internacional sobre Nutrición y Deporte, Barcelona, 1999 a.

MARINS, J. C. B.; MARINS, N. M. O.; VILLEGAS, J.; ZAMORA, S. Hábitos de hidratación en un colectivo de triatletas, Curso Internacional sobre Nutrición y Deporte, Barcelona, $1999 \mathrm{~b}$

MARINS J. Estudio comparativo de diferentes procedimientos de hidratación durante un ejercicio de larga duración. Tesis Doctoral.: Departamento de Fisiología y Farmacología. Universidad de Murcia; 2000.

MARINS, J.; DANTAS, E.H.; ZAMORA NAVARRO, S. Deshidratación y ejercicio físico. Selección 20009 (3): 33 - 47

MARINS, J.C.B.; AGUDO, C.; IGLESIAS, M.L.; MARINS, N.; ZAMORA, S. Hábitos de hidratación en un colectivo de deportistas de pruebas de resistencia. Seleción 13(1):1828,2004

MORI, S. OHTANI, Y. IMANAKA, K. Reaction times and anticipatory skills of karate athletes. Humam Mov. Sci. 21:213-30, 2002

NIEUWENHOVEN M. A.; BRUMMER, R. J. M.; BROUNS, F. Gastrointestinal function during exercise: comparison of water, sports drink and sports drink with caffeine, J. Appl. Physiol. 89: 1079-1085, 2000

OHTA, S.; NAKAJI, S.; SUZUKI, K.; TOTSUKA, M.; UMEDA, T.; SUGAWARA, K. Depressed humoral immunity after weight reduction in competitive judoists. Luminescence 2002; $17(3): 150-7$.

ROEMMICH, J.N.; SINNING, W.E. Weight loss and wrestling training: effect of growthrelated hormones. J. Appl. Physiol. 82(6):1760-4, 1997.

SAUNDERS, P.U.; WATT, M.J.; GARNHAM, A.P.; SPRIET, L.L.; HARGREAVES, M.; FEBBRAIO, M.A. No effect of heat mild stress on regulation of carbohydrate metabolism at the onset of exercise. J. Appl. Physiol. 91: 2282-8, 2001.

SOPER, J.; CARPENTE, R.; SHANNON, B. Nutrition knowledge of aerobic dancers instructors. J. Nutr. Educ. 24(2):59-65, 1992.

STRACHAN, A.T.; LEIPER, J.B.; MAUGHAN, R.J. Serotonin2c receptor blockade and thermoregulation during exercise in the heat. Med. Sci. Sports Exerc. 37(3):389-94, 2005

SUZUKI, M.; NAKAJI, S.; UMEDA, T.; SHIMOYAMA, T.; MOCHIDA, N.; KOJIMA, A.; MASHIKO, T.; SUGAWARA, K.. Effects of weight reduction on neutrophil phagocytic activity and oxidative burst activity in female judoists. Luminescence 2003; 18(4):214-7.

WALTON, C.; KALMAR, J.M.; CAFARELLI, E. Effect of caffeine in self-sustained firing in human motor units. J. Physiol. 2002; 554.2:671-9.

WINNICK, J.J.; DAVIS, J.M.; WELSH, R.S.; CARMICHAEL, M.D.; MURPHY, E.A.; BLACKMON, J.A. Carbohydrate Feedings during Team Sport Exercise Preserve Physical and CNS Function. Med. Sci. Sports Exerc. 37(2):306-15, 2005

\section{QUESTIONÁRIO PARA VERIFICAR O NÍVEL dE CONHECIMENTO SOBRE HIDRATAÇÃO}

\section{Sexo:}

[ ] Masculino

[ ] femenino

\section{Idade:}

[ ]

\section{Categoría de peso:}

1 - Quantos anos você tem de prática no esporte como atleta?<smiles>C=C[Tl]</smiles>

2 - Você tem o costume de hidratar-se durante:

\begin{tabular}{|c|c|c|c|c|c|c|c|}
\hline \multicolumn{4}{|c|}{ Durante o treinamento } & \multicolumn{4}{|c|}{ Durante competições } \\
\hline Nunca & $\begin{array}{l}\text { Quase } \\
\text { nunca }\end{array}$ & $\begin{array}{c}\text { Às } \\
\text { vezes }\end{array}$ & Sempre & Nunca & $\begin{array}{l}\text { Quase } \\
\text { nunca }\end{array}$ & $\begin{array}{c}\text { Às } \\
\text { vezes }\end{array}$ & Semp \\
\hline & & & & & & & \\
\hline
\end{tabular}

3 - Quando você se hidrata, seu costume é:

\begin{tabular}{|c|c|c|c|c|c|}
\hline \multicolumn{2}{|l|}{ Durante o treinamento } & \multicolumn{3}{|c|}{ Durante competições } \\
\hline Antes & Durante & Depois & Antes & Durante & Depois \\
\hline & & & & & \\
\hline
\end{tabular}

4 - Quando você se hidrata, se preocupa com o tipo de hidratação (água ou bebida carboidratada) nos momentos que antecedem, durante ou depois de um treinamento e competição? [ ] Sim

[ ] Não

5 - Qual o tipo de solução que você consome em cada momento?

\begin{tabular}{|c|c|c|c|}
\hline Solução & Antes & Durante & Depois \\
\hline Água & & & \\
\hline Bebida Carboidrata & & & \\
\hline
\end{tabular}

6 - Quando se deve beber líquidos?

[ ] Antes da sensação de sede

[ ] Somente depois de sentir sede

[ ] Quando se sente muita sede

7 - Qual o tipo de solução líquida que você tem o costume de se hidratar?

[ ] Agua [ ] Bebida Carboidrata [ ] Refrescos
[ ] Sucos naturais
$\left.\begin{array}{ll}\text { [ ] Café } & \text { [ ] Coca-cola }\end{array}\right]$


8 - Qual o tipo de bebida carboidratada você conhece?

[ ] Não conheço nenhum [ ] Marathon [ ] Energil C

[ ] SportAde [ ] Sportdrink [ ] Gatorade

[ ] Red Bull [ ] Otros

9 - Entre as bebidas carboidratadas que você conhece, qual a de sua preferência?

[ ] Marathon [ ] Energil C [ ] SportAde
[ ] Sportdrink [ ] Gatorade [ ] Red Bull
[ ] Outros

10 - Qual o sabor de bebida carboidratada que você mais gosta?

$\begin{array}{lll}\text { [ ] Laranja [ ] Tangerina } & \text { [ ] Uva } \\ \text { [ ] Limão } & \text { [ ] Maracujá } & \text { [ ] Frutas cítricas } \\ \text { [ ] Outros } & \end{array}$

11 - Sua preocupação quanto à necessidade de se hidratar é mais freqüente:

[ ] No verão [ ] No inverno [ ] Independente da estação

[ ] Não me preocupo

12 - Você tem o costume de pesar-se antes e depois de um treinamento ou competição?

[ ] Sim, frequentemente [ ] Sim, mas não frecuentemente
[ ] Quase nunca

13 - Durante uma competição ou treinamento, você já apresentou algum destes sintomas?

[ ] Sede muito intensa

[ ] Câimbras

[ ] Dificuldade de concentração

[ ] Palidez

[ ] Desmaios

[ ] Olho fundos

[ ] Insensibilidade das mãos

[ ] Sensação de perda de força

[ ] Fadiga generalizada [ ] Dor de cabeça

[ ] Alucinações [ ] Sonolencia

[ ] Perda momentanea da consciência

[ ] Convulsões [ ] Coma

[ ] Interrupção da prodrução de suor

[ ] Interrupção da atividade planificada

[ ] Dificuldade de realizaçao de um movimeno técnico, fácilmente realizado em condições normais
14 - Como que você acha que deveria ser feita uma hidratação:

[ ] Beber um litro de uma só vez

[ ] Beber $1 / 4$ litro para cada $1 / 4$ de hora

[ ] Beber $1 / 2$ litro para cada $1 / 2$ hora

[ ] Não tenho idéia

15 - Você se preocupa com o tipo de roupa que utiliza durante o exercício, além do equipamento da modalidade?

[ ] Sí

[ ] No

Em caso afirmativo responda a seguinte pergunta: Qual a sua preocupação?

[ ] Cor [ ] Tipo de tecido

[ ] Qantidade de tecido

16 - Quando você se hidrata a temperatura de líquido costuma ser:

[ ] Extremadamente gelado

[ ] Moderadamente gelado

[ ] Temperatura normal

17 - Você já teve alguma orientação sobre qual a melhor maneira de se hidratar?

[ ] Sí

[ ] No

* Em caso afirmativo: Quem prestou a orientação?

[ ] Médico [ ] Fisioterapeuta

[ ] Livros [ ] Preparador Físicos

[ ] Diretor de equipe [ ] Revistas

[ ] Técnico [ ] Prof. de Ed. Física da escola

[ ] Amigos [ ] Treinador

[ ] Pais [ ] Outros

[ ] Nutricionista

18 - Você acredita que o consumo de uma bebida carboidratada:

[ ] Repõe só líquidos [ ] Repone só eletrólitos

[ ] Repõe só energia [ ] Repõe só eletrólitos

[ ] Hidrata e repõe eletrólitos e energia

[ ] Apresenta a mesma funçao da hidratação com agua 